\title{
Sarcoidosis - Three Clinical Vignettes with a Short Review
}

\author{
Kishore PV, ${ }^{1}$ Paudel R, ${ }^{1}$ Paudel B, ${ }^{1}$ Palaian S, ${ }^{2}$ Mishra P' \\ 'Department of Medicine, ${ }^{2}$ Department of Clinical Pharmacy, Manipal College of Medical Sciences \\ Pokhara, Nepal.
}

\begin{abstract}
Sarcoidosis is a multi system disorder of unknown cause most commonly affecting the young and middle age adults and frequently presents bilateral hilar lymph-adenopathy. The diagnosis of sarcoidosis is often delayed following the onset of symptoms. The reason being first, the disease is often sub clinical; second as the disease affects any system, the diagnosis of sarcoidosis may not be considered; third, the symptoms are not disease specific hence often treated as other chronic pulmonary diseases; finally economic issues or barriers to access for further workup may affect the timeliness of the diagnosis. No laboratory diagnosis is specific for diagnosing sarcoidosis but histological confirmation from the lymph nodes accessible for biopsy either direct or by intervention may be more specific.
\end{abstract}

Key words: Corticosteroids, Lymph node, Sarcoidosis.

\section{INTRODUCTION}

We describe here three different short case reports with varied presentations diagnosed by exclusion and supportive evidence. Only the first case report mentioned was histo pathologically confirmed, but the rest of the cases are diagnosed basing on supportive evidence, common presentation and excluding other granulomatous diseases. Since, the lesions are not easily accessible for histo pathological confirmation in resource poor centers, a high degree of suspicion and an approach of exclusion with supportive evidence would be helpful for the treating physician to initiate the therapy.

\section{CASE REPORT 1}

A 54 year male presented with dry cough, shortness of breath and tender erythematous nodules over both legs with no history of fever. On investigation found to be having high Erythrocyte sedimentation rate (ESR) and
Chest radiograph, showing bilateral hilar opacities with reticulo-nodular pattern. Pulmonary Function Tests (PFT) was of restrictive pattern with negative tuberculin test. After three weeks of initial symptoms, he developed left forearm abscess as suggested by an ultra sonogram. Abscess was drained and pus was negative for acid-fast bacilli (AFB) and other microorganisms. Again After six weeks he developed swelling at middle of left little finger with discharging sinus, radiograph revealed resorption of the middle phalanx of the little finger for which incision and drainage was done and biopsy of the excised tissue revealed non-caseating granulomas. Pus was negative for AFB and other microorganisms. Based on these observations a clinical diagnosis of sarcoidosis with multisystem involvement was made and started on oral corticosteroid therapy. The patient improved both clinically and radiologically and was on regular follow up.

\author{
Correspondence: \\ Dr. P.V. Kishore \\ Department of Medicine \\ Manipal College of Medical Sciences \\ Pokhara, Nepal. \\ Email:pandu_vki@yahoo.com
}




\section{CASE REPORT 2}

A 37 year woman presented with three days history of sudden weakness of left side of the face with slurred speech otherwise asymptomatic. She found to have bilateral hilar opacities in her chest radiograph two months back when she had to undergo hysterectomy and started on anti-tuberculosis therapy empirically. On investigation, her ESR was high, computed tomography (CT) scan of the brain was normal and her latest chest radiograph showed increase in the size of the hilar opacities on both sides. Her tuberculin test was negative. She was diagnosed to have sarcoidosis (Stage-I) with facial nerve palsy and was treated successfully with oral corticosteroids.

\section{CASE REPORT 3}

A 50 year male presented with bilateral lower motor neuron facial paralysis with the rest of the systems being normal. On investigation, he has raised ESR, normal CT scan of the brain, but bilateral hilar opacities in chest radiograph and $\mathrm{CT}$ scan of the thorax. He also had negative tuberculin test. Based on all these findings a clinical diagnosis of sarcoidosis associated with bilateral facial nerve palsy was made. The patient was subsequently treated with oral corticosteroids with a good response.

\section{THE CLINICAL PROBLEM}

Many diseases may have a hilar adenopathy both unilateral and bilateral. The diagnostic work up of a patient with hilar adenopathy with multi system or remote extra pulmonary areas involvement and the possible diagnosis of Sarcoidosis depends upon the proper exclusion of other common chronic diseases and a sense of high suspicion. The diagnosis of Sarcoidosis, is often delayed following the onset of symptoms. Several reasons exist for the delay. First, the disease is often sub-clinical or self-limiting, or the symptoms are minimal. Screening chest radiograph surveys have suggested that a large proportion of pulmonary sarcoidosis cases remain undiagnosed because patients are asymptomatic. Second, the disease can affect any organ system, for which are referred to a wide range of specialists, some of whom may not consider sarcoidosis in the differential diagnosis. Third, the symptoms of sarcoidosis are not specific and often suggest alternative pulmonary diseases. Finally, economic issues or barriers to access to medical care may affect the timeliness of diagnosis. The diagnosis of Sarcoidosis is made on the first physician visit in only $15.3 \%$ of cases, and slightly less than one-half of the cases required four or more physician visits until the diagnosis of Sarcoidosis is made. ${ }^{1}$

\section{SARCOIDOSIS}

In 1991, members of the World Association of Sarcoidosis and other Granulomatous Disorders proposed, "Sarcoidosis is a multisystem disorder of unknown cause. It most commonly affects young and middle-aged adults and frequently presents with bilateral hilar adenopathy, pulmonary infiltration, and ocular and skin lesions. Liver, spleen, lymph nodes, salivary glands, heart, nervous system, muscles, bone and other organs may also be involved. The diagnosis is established when clinicoradiological findings are supported by histologic evidence of non-caseating epitheloid cell granulomas. Granulomas of known causes and local sarcoid reactions must be excluded". 2

\section{EPIDEMIOLOGY}

The disease occurs worldwide and may be seen at any age, but the disease most commonly recognized in patients between the ages of 20 and 40 years. ${ }^{3} \mathrm{~A}$ second peak in incidence occurs in women older than 50 . There is considerable variation in the reported incidence and prevalence in different countries and continents. Disease is particularly common in African Americans, especially women, and particularly rare in Chinese. One prospective study from Eastern India has reported 125 cases of biopsy proven sarcoidosis. The authors claimed that the presentation, clinical course and radiological features are considerably different from those seen in the Western countries. ${ }^{4}$ In general, in countries like India the prevalence of sarcoidosis is considered low. The lower prevalence is possibly associated with the absence of mass chest radiographic screening and due to the presence of other, more commonly recognized granulomatous diseases, especially tuberculosis, that mimic sarcoidosis. 5 In a study conducted in Nepal, 33 patients were subjected to trans-bronchial needle aspiration during the routine diagnostic bronchoscopy. Among these patients, one of them had sarcoidosis. ${ }^{6}$

\section{ETIOLOGY AND PATHOGENESIS}

The etiology and pathogenesis of the disease are unclear and undoubtedly complex. The recent data suggest that immunologic mechanisms involving cytokine-producing $\mathrm{CD}^{+}{ }^{+} \mathrm{T}$-cell helper 1-lymphocytes and mononuclear phagocytes may be responsible for the lung damage and for the formation of 'noncaseating granulomas'-the pathologic hallmark of sarcoidosis. ${ }^{10}$ 


\section{CLINICAL MANIFESTATIONS}

The clinical manifestations are varied in sarcoidosis, reflecting its multisystem involvement. Pathologically, the most commonly involved organ systems are the lungs $(90 \%)$, peripheral lymphatics $(50 \%-75 \%)$, skin (10\%-30\%), liver (60\%-80\%), eye (17\%), spleen (15\%), bone $(1 \%-35 \%)$, salivary glands $(5 \%)$, joints $(10 \%-$ $15 \%)$, and heart (30\%). ${ }^{7}$ Clinical characteristics occur at a much slow rate, because sarcoid lesions may be present in some organs without affecting their function. Cough and dyspnea are the most common respiratory complaints. Erythema nodosum is seen occasionally with the typical presentation of bilateral hilar adenopathy and connotes a good prognosis. Approximately $30 \%$ of patients are asymptomatic and only an abnormal chest $\mathrm{x}$-ray identifies it. Constitutional symptoms are common and include weight loss, fatigue, weakness, and malaise. Fever occurs in $15 \%-20 \%$ of the patients. ${ }^{7}$

The cardiovascular system can be affected directly or indirectly. Indirect involvement is manifested by pulmonary arterial hypertension and cor pulmonale and can be caused by parenchymal fibrosis or hypoxemia. Direct involvement is seen in $25 \%$ of cases at autopsy. The presence of arrhythmias or unexplained heart failure in a young person should suggest the possibility of myocardial sarcoidosis. ${ }^{8}$

Enlargement of peripheral lymph nodes is clinically evident in $75 \%$ of cases, more so in the cervical region. ${ }^{10}$ The characteristic lesion in the eye is Uveitis, but other structures also may be involved. The most frequent non specific abnormality is Erythema Nodosum, a form of panniculitis most commonly involving the shins which are transient, non ulcerating, tender, multiple and bilateral nodules.

Granulomas may be found in liver and spleen at autopsy in most of the cases but palpable enlargement is seen in only few and may mimic any primary diseases of the respective organs. Symptomatic involvement of gastrointestinal tract is very rare. Involvement of minor salivary glands has been noted in almost $60 \%$ of random lip biopsies in one study. ${ }^{9}$ The combination of parotid gland involvement and uveitis with pyrexia is called as uveoparotid fever and if it is with Facial nerve palsy, it is termed as Heerfordt's syndrome.

Three forms of joint involvement have been described: 1) migratory polyarthritis associated with Erythema nodosum, fever, and hilar lymph node enlargement (most common) called as Lofgren's Syndrome; 2) single or recurrent episodes of polyarticular or monoarticular arthritis; and 3) persistent arthritis. Clinical or functional evidence of renal diseases uncommon but granulomas may be found in the kidneys in 5\%-20\% at autopsy. ${ }^{3}$

Involvement of the nervous system is evident in $5 \%$ of patients during life and in 15\%-25\% at autopsy. ${ }^{3}$ Abnormalities may be related to the cranial and peripheral nerves, the brain, the spinal cord, and the meninges. Although any cranial nerve can be affected, the second and seventh are most commonly involved. Unilateral or bilateral facial palsy is sometimes associated with uveoparotid fever.

\section{RECOMMENDED CLINICAL EVALUATION IN SARCOIDOSIS}

The diagnosis of sarcoidosis is based on the parameters like the presence of a compatible clinical picture, the exclusion of other Granulomatous disease and histological evidence of noncaseating granulomas on tissue biopsy. The histologic picture is not pathognomonic, and tuberculosis, fungal disease, beryllium disease, drug reaction, and local sarcoid reactions must be excluded.

The American Thoracic Society (ATS) recommendations for comprehensive initial evaluation include the following. ${ }^{10}$

- History, including occupational and environmental exposure

- Physical examination

- Postero-anterior chest radiography

- Pulmonary function tests, including spirometry and diffusing capacity of the lung for Carbon Monoxide (DLCO)

- Peripheral blood counts

- Serum chemistries, including creatinine, calcium and liver enzymes

- Urine analysis

- Electrocardiogram

- Routine ophthalmologic examination

- Tuberculin skin test

Chest $\boldsymbol{X}$-Ray The sarcoidosis when involving lung is staged as: Stage 0, Clear chest $x$-ray (5\%-16\%); Stage 1 , bilateral hilar adenopathy (25\%-40\%); Stage 2, bilateral hilar adenopathy with pulmonary infiltrate (24\%-49\%); and stage 3, pulmonary infiltrates without hilar adenopathy $(6 \%-15 \%)^{7}$

Pulmonary function tests reveal restrictive abnormalities plus measurement of gas exchange showing reduction in diffusing capacity of carbon monoxide. 
Kishore et al. Sarcoidosis - Three Clinical Vignettes with a Short Review

Ophthalmologic evaluation with slit-lamp examination has to be performed.

Laboratory Tests are generally not helpful in making a diagnosis. No definitive diagnostic blood, skin, or radiological imaging tests are specific for this disorder. Angiotensin-l-converting enzyme (ACE) levels are increased in $60 \%-80 \%$ of patients with sarcoidosis but this finding is not specific. ${ }^{7}$ This test should be interpreted, in light of the other clinical conditions in which it can be elevated; Serum calcium levels may be increased in some; other tests depending on clinical presentation and suspicion of extra thoracic disease are performed for assessing the extent and severity of organ involvement.

Tuberculin skin test is often negative and aids in initial evaluation of sarcoidosis apart from other granulomatours diseases like tuberculosis. In one study, $73 \%$ of the sarcoidosis patients has a negative tuberculin skin test. However, but the number of positive cases was still substantial $(27 \%) .{ }^{11}$

Gallium-67 scanning is a noninvasive radioisotope technique that reflects, to a degree, the activity of the lung involvement in sarcoidosis. The pattern of radioactive highlighting of bilateral hilar and right Paratracheal lymph nodes has been termed as lamda sign; the highlighting of parotid, lacrimal, and salivary glands, the panda sign; the presence of both lamda and panda signs are somewhat specific for sarcoidosis. As with measurements of ACE levels, even the Gallium scanning is nonspecific as it is abnormal in other conditions as well.

Bronchoalveolar lavage (BAL) fluid demonstrates increased numbers of CD4 T-helper lymphocytes, which also carries no specificity.

Kveim-Siltzbach skin test spleen or lymph-node homogenate from a patient with sarcoidosis is injected intradermally and later subjected to biopsy. It is not well standardized, and not approved for general use by the United States Food and Drug Administration (US FDA).

Biopsy is done to obtain histologic confirmation of noncaseating granulomas. Biopsy of an abnormal lymph node, parotid gland, nasal mucosal lesion, or other involved sites usually yield the diagnosis. Other methods of tissue diagnosis are transbronchial lung biopsy $191 \%$ yield), mediastinoscopy (96\%), open lung biopsy $(100 \%$ yield), liver biopsy (70\%), blind scalene-node biopsy (40\% - 70\%), blind bronchial mucosal biopsy (50\%), and blind subconjunctival biopsy (50\%). The selection of any of these procedures should be guided by the clinical presentation, with careful explanation of the risks and benefits to the patient. ${ }^{7}$

\section{INDICATIONS FOR TREATMENT}

Most patients $(\sim 80 \%)$ require no therapy. There are no strict guidelines regarding indications for treatment of sarcoidosis. However, one review recommend that asymptomatic patients with bilateral hilar lymphadenopathy (Stage I) with or without erythema nodosum but without extrapulmonary involvement should be left untreated, patients with bilateral hilar adenopathy and infiltration (Stage II) and symptoms (cough, dyspnoea, chest pain, exercise intolerance) should be treated with corticosteroids. Similarly, patients with Stage III disease with diffuse pulmonary infiltration usually have symptoms along with lung function abnormalities and almost always need treatment and the stage IV diseases with extensive fibrosis and bullae formation respond poorly or not at all to corticosteroids and immunosuppressive therapy. ${ }^{12}$

\section{DRUG TREATMENT}

Two recent studies, The British Thoracic Society study and the meta-analysis of corticosteroid therapy in pulmonary sarcoidosis study, support a beneficial role in the management of sarcoidosis. ${ }^{13}$ Symptomatic pulmonary disease cases may require only observation, but may be considered for oral followed by inhaled steroids if recently diagnosed stage II disease. Stage II or III pulmonary diseases with symptoms and/or abnormal function, and severe extra pulmonary disease, including heart, central nervous system, eyes, kidneys has to be treated with systemic steroids. Although there is evidence to support benefit from corticosteroid therapy, the issues of patient selection, timing of initiation, and duration of therapy remain unresolved. There is also no consensus regarding the ideal dose. Suggestions range from $40 \mathrm{mg}$ of prednisone every other day to $1 \mathrm{mg} / \mathrm{kg}$ body weight daily. However, mostly the response to $30-40 \mathrm{mg}$ of prednisone is evaluated for 4 - 12 weeks, patients who do not improve within this period are unlikely to respond, and prednisone should be slowly tapered in them. It is recommended that steroid responders continue therapy for a total of 12 - 18 months before discontinuing steroids. In individuals who have had continued deterioration despite use of corticosteroids or who cannot tolerate the corticosteroid side effects, alternate therapy is used. Methotrexate is the first alternate often used.

About $10 \%$ of the patients requiring therapy are unresponsive to a tolerable doses of a corticosteroid and should be given a 6 months trial of methotrexate starting at $2.4 \mathrm{mg} /$ week orally and increasing in increments of $2.5 \mathrm{mg} /$ week to a total of $10-15 \mathrm{mg} /$ week as tolerated by a WBC $>3000 / \mathrm{mm}^{3}$. After eight week of methotrexate, 
the orticosteroid can be reduced and then often discontinued. Serial blood counts and liver enzyme tests should be performed every 6 week. ${ }^{14}$

Hydroxychloroquine $200 \mathrm{mg}$ twice daily is more effective than corticosteroids for treatment of disfiguring skin sarcoids. Retinal damage is rare, but serial ophthalmologic examination should be performed every 6 months. ${ }^{14}$

Azathioprine has a role in the treatment of sarcoidosis. One review article reported it to be a reserved drug in neurosarcoidosis. The author recommends a relatively low dose of $50 \mathrm{mg}$ per day during the initiation of therapy to determine the patient's sensitivity to azathioprine within the first month. The patients should be monitored every month for complete blood counts and renal function tests. ${ }^{12}$ In one study of 21 patients the patients responded well with the drug and was associated with an improvement of both pulmonary and extrapulmonary disease. Eleven patients had a relapse with withdrawal of drug. In all cases, the patients responded to reinstitution of azathrioprine. ${ }^{15}$

There are anecdotal reports of Cyclophosphamide use in sarcoidosis. However, it is the most effective agent for patients with advanced stages of Wegener's granulomatosis. ${ }^{10}$

While using cyclopbhosphamide, one should be aware of its cytotoxic potential similar to that of any other cytotoxic drugs. It can cause hemorrhagic cystisis, alopecia etc.

Other agents that are used are, chloroquine or hydroxychloroquine, chlorambucil, pentoxyfylline, thalidomide, and cyclosporin, but there is no supporting evidence favoring these agents. ${ }^{15}$

Transplantation is the last option for patients who fail to respond to medical therapy. Successful lung, heartlung, heart, liver, and kidney transplants have been performed and lung and liver transplants are reported with similar survival in both sarcoid and nonsarcoid patients.

\section{CONCLUSION}

Sarcoidosis is a multi system disease most commonly involving the lungs, mimicking other chronic granulomatous disorders and poses a diagnostic challenge to the treating physician. The diagnosis of sarcoidosis isbased on the presence of a compatible clinical picture, the exclusion of other granulomatous diseases and histological evidence of noncaseating granulomas on tissue biopsy. When once diagnosed, the patient may be kept under observation ( $80 \%$ of the cases undergo spontaneous remission) or may be initiated on corticosteroid therapy according to the severity of the illness, to avoid the long-term dysfunction of the affected organs.

\section{REFERENCES}

1. Judson MA, Thompson BW, Rabin DL, et al. The diagnostic pathway to sarcoidosis. Chest. 2003;123(2):406-12.

2. Yamamoto M, Sharma OP, Hosoda Y. Special report: the 1991 descriptive definition of sarcoidosis. Sarcoidosis 1992;9(Suppl 1):33-4.

3. Fraser RS, Neilcolman, Muller NL, Pare PD. Synopsis of diseases of the chest. 3rd ed. New Delhi: Elsevier Publishers;2006. p.445-80.

4. Gupta SK, Gupta S. Sarcoidosis in India: a review of 125 biopsy-proven cases from eastern India. Sarcoidosis 1990 Mar;7(1):43-9.

5. Shetty A, Gedalia A. Sarcoidosis [Internet]. USA: emedicine, webMD; [updated 2006 Jun 9; cited 2006 Dec 8];[11 screens]. A v a i l a b l e f $\mathrm{r}$ o m : U R L : http://www.emedicine.com/PED/topic2043.htm

6. Chokhani R. Transbronchial needle aspiration in the diagnosis of respiratory diseases. Nepal Med Coll J 2004;6(1):24-7.

7. Bordow RA, Ries AL, Morris TA. Manual of Clinical problems in Pulmonary Medicine. Philadelphia: Lippincott Williams Wilkins;2001.

8. Silverman KJ, Hutchins GM, Bulkley BH. Cardiac sarcoid: a clinicopathologic study of 84 unselected patients with systemic sarcoidosis. Circulation. 1978 Dec;58(6):1204-11.

9. Nessan VJ, Jacoway JR. Biopsy of minor salivary glands in the diagnosis of sarcoidosis. N Engl J Med 1979;301(17):922-4.

10. American Thoracic Society. Statement on sarcoidosis [internet]. New York: American Thoracic Society; [cited 2006 Dec 8]. Available from: URL: http://www.thoracic.org/sections/publications/statement s/pages/respiratory-disease-adults/sarcoid1-20.html

11. Chevalet P, Clement R, Rodat O, Moreau A, Brisseau JM, Clarke JP. Sarcoidosis diagnosed in elderly subjects: retrospective study of 30 cases. Chest 2004;126(5):1423-30.

12. Sharma OP. Pulmonary sarcoidosis: management. J Post Grad Med 2002;48:135-41.

13. Wasfy Y, Fontenot AP. Sarcoidosis. In: Current diagnosis and treatment in pulmonary medicine, Hanly $\mathrm{ME}$, Welsh $\mathrm{CH}$, editors. New Delhi: Tata McGraw-Hill publishing company;2005. p.131-8.

14. Beers MH, Berkow R, Editors. The Merck Manual. 17th ed. White House Station: Merck Research Laboratories; 1999. p.453-4.

15. Hof DG, Hof PC, Godfrey WA. Long-term use of azathioprine as a steroid-sparing treatment for chronic sarcoidosis. Am J Respir Crit Care Med 1996;153:A870. 